\title{
Serum Neudesin Levels in Obese Adolescents
}

\author{
(1) Aliye Çelikkol1, (1) Çiğdem Binay², (1) Özge Ayçiçek ${ }^{1}$, (1) Savaş Güzel1 \\ 1 Tekirdağ Namık Kemal University Faculty of Medicine, Department of Medical Biochemistry, Tekirdağ, Turkey \\ ${ }^{2}$ Tekirdağ Namık Kemal University Faculty of Medicine, Department of Pediatric Endocrinology, Tekirdağ, Turkey
}

\section{What is already known on this topic?}

Neudesin is a neurotropic factor that contributes to the complex control of energy homeostasis. In addition, neudesin can influence appetite control in the hypothalamus or the anxiety-like behaviour controlled by the dentate gyrus of the hippocampus.

\section{What this study adds?}

We assessed the relationship between obesity, metabolic complications and neudesin levels in adolescents. Neudesin levels were significantly decreased in obese and morbidly obese adolescents. Neudesin may have a significant potential role in the regulatory mechanisms of obesity and other metabolic disorders.

\begin{abstract}
Objective: Advances in knowledge of neurotrophic factors are now revealing the complex control of energy homeostasis and appetite, as well as the crucial role these factors play in nervous system function. The aim of this study was to assess serum levels of neudesin in adolescents with obesity and to examine the relationship between these levels and metabolic outcomes.

Methods: Adolescents, aged 10-17 years were enrolled. Subjects were divided into normal weight, obese and morbidly obese subgroups. Serum neudesin concentrations were compared between the groups.

Results: In total, 88 adolescents were recruited, of whom 30 (34.1\%) were normal weight, 15 (17.0\%) were obese and 43 (48.9\%) were morbidly obese. Neudesin levels were significantly lower in obese adolescents than in the control group $(p=0.013)$. A correlation analysis applied to the whole study group revealed a negative correlation between serum neudesin concentration and body mass index (BMI) $z$ scores $(r=-0.40, p<0.001)$. Serum neudesin levels tended to increase in adolescents with metabolic syndrome, insulin resistance, dyslipidaemia, and hypertension but the differences were not significant $(p=0.259, p=0.246, p=0.259$, and $p=0.523$, respectively).

Conclusion: Serum neudesin levels were significantly correlated with BMI z score in obese adolescents. Generally, serum neudesin levels were low in obese and morbidly obese adolescents and tended to increase with the appearance of metabolic disorders. Both obesity and associated metabolic disorders have multifactorial causes. Therefore, we suggest that the role of the neudesin molecule in the regulatory mechanisms of obesity and metabolic disorders should be further investigated with well-designed studies enrolling larger groups.
\end{abstract}

Keywords: Obesity, neudesin, insulin resistance, metabolic syndrome

\section{Introduction}

Obesity in children and adolescents has increased worldwide in the last 30 years. The most important reasons for this increase are the decrease in physical activity in children and the negative effects of wider use of developing technology and poorer eating and drinking habits (1). The epidemic of obesity is closely associated with an increase in metabolic disorders, including diabetes, dyslipidemia and cardiovascular diseases, and this has prompted major interest in the regulation of adipose functions.

Adipose tissue can be divided into two distinct types: white and brown. White adipose tissue (WAT) is specialised for the storage of excess energy as triglycerides, whereas brown adipose tissue (BAT) dissipates energy as heat, thereby counteracting obesity. The regulation of adipose tissue function depends on the sympathetic nervous system 
(SNS), which plays a fundamental role in maintaining energy homeostasis in living organisms (2). The SNS also modulates the development of obesity because it stimulates lipolysis in WAT and enhances heat production in BAT by activating adrenergic signalling (3).

The functioning of the SNS depends on neurotrophic factors which promote the survival, differentiation and maintenance of neurons in the vertebrate nervous system $(4,5,6)$. One newly identified neurotropic factor is neudesin, a compound that contributes to the complex control of energy homeostasis. Human neudesin is a 172 amino acid protein that shares a high sequence similarity to neudesin found in other vertebrates. Neudesin activates the mitogen-activated protein kinase and phosphoinositide 3-kinase signalling pathways and has a role in neural cell differentiation, cell proliferation and tumorigenesis (7). Neudesin is preferentially expressed in the central nervous system and the spinal cord, where it promotes neural cell differentiation. Neudesin mRNA expression has also been documented in other tissues, including adipose tissue, heart, lungs, and kidney. In addition to its neurotropic effects, neudesin can influence appetite control in the hypothalamus or the anxiety-like behaviour controlled by the dentate gyrus of the hippocampus (3).

Neudesin has been examined in only a limited number of studies to date, but the current consensus is that neudesin may have potential for the treatment of obesity and obesityrelated disorders $(3,7)$. The aim of the present study was to investigate the levels of circulating serum neudesin in a group of adolescents with obesity and to examine the relationship between these levels and metabolic outcomes that develop due to obesity.

\section{Methods}

\section{Participants}

This prospective study included adolescents aged 10 to 17 years. Of these adolescents, around two thirds were referred to the paediatric endocrinology outpatient clinic because of weight gain and were diagnosed with obesity. The other third were healthy age- and sex-matched children who served as the control group. Exclusion criteria were: chronic or hereditary diseases; endocrinological disorders including syndromes associated with obesity; and a history of drug use. Pubertal stage was assessed according to the criteria described by Marshall and Tanner (8).

A challenge in determining the prevalence of metabolic syndrome (MS) is the existence of multiple definitions and criteria used to identify this condition. In response, the International Diabetes Federation (IDF) released the IDF Consensus Worldwide Definition of MS as a single, universally accepted tool. The IDF defines MS in children and adolescents as the presence of abdominal obesity (waist circumference $\geq 90^{\text {th }}$ percentile by age and sex) and the presence of two or more of the following clinical features: an elevated triglyceride level $(\geq 1.7 \mathrm{mmol} / \mathrm{L})$, a low high-density lipoprotein cholesterol (HDL-C) level $(<1.03 \mathrm{mmol} / \mathrm{L})$, high blood pressure (systolic blood pressure $\geq 130 \mathrm{mmHg}$ and/or diastolic blood pressure $\geq 85 \mathrm{mmHg}$ and/or a diagnosis of hypertension) and an elevated glucose level $(\geq 5.6 \mathrm{mmol} / \mathrm{L}$ and/or a diagnosis of type 2 diabetes) (9). Written informed consent was obtained from the children's parents after they were informed of the aim and procedures of the study. The study was approved by the Tekirdağ Namık Kemal University University of Non-Invasive Clinical Research Ethics Committee (protocol number: 2019.137.08.09, date: 01.08.2019).

\section{Study Design}

The height of each participant was measured using a wall-mounted stadiometer sensitive to the nearest 0.1 $\mathrm{cm}$ (Harpenden, Holtain, Crymych, UK) and with the adolescent in a standing position, without shoes. Weight was measured using a portable calibrated scale sensitive to the nearest $0.1 \mathrm{~kg}$ (SECA762; Voge \& Hakle, Hamburg, Germany), with the participants wearing light clothing. The body mass index (BMI) was calculated as weight (kg) divided by height $\left(\mathrm{m}^{2}\right)$. The height, weight and BMI were expressed as standard deviation scores (SDS) using the 2007 growth reference percentiles for Turkish children and adolescents (10). Children were divided into three groups - normal weight, obese, or morbidly obese (11). Blood pressure was measured using an automated sphygmomanometer. Elevated blood pressure ( $295^{\text {th }}$ percentile for height) was determined using tables provided by the Task Force Report (12).

\section{Statistical Analysis}

The Kolmogorov-Smirnov test was used to assess normality of data distribution from the control and patient groups. Parameters were evaluated according to their nonparametric and parametric distributions. Differences between different groups were assessed using the Student's t-test for parametric data, while the Mann-Whitney $U$ test was used for data with nonparametric distribution. Relationships between parameters were investigated using Pearson correlation analysis. The correlations were modelled by linear regression analysis of different sizes and were found to be similarly significant. All statistical analyses were performed with the Statistical Package for the Social 
Sciences, version 22.0 (IBM Inc., Armonk, NY, USA). A p value of $<0.05$ was considered statistically significant.

\section{Laboratory Measurements}

In both the patient and control groups, peripheral venous blood samples were collected after 12-hour overnight fasting and used for enzymatic measurements of the concentrations of glucose, insulin, free thyroxine, thyroidstimulating hormone, low-density lipoprotein cholesterol (LDL-C), HDL-C, triglycerides, total cholesterol (TC) and alanine aminotransferase (Roche Modular DP Automatic Biochemical Analyser; Roche Diagnostics, Indianapolis, IN, USA). We used the homeostasis model assessment of insulin resistance (HOMA-IR) to determine the presence of IR by employing the following formula: fasting glucose ( $\mathrm{mmol} / \mathrm{L}$ ) $\times$ fasting insulin (IU/L)/22.5. The HOMA-IR cut-off values for IR were taken as 5.22 in boys and 3.82 in girls (13).

\section{Results}

In total, 58 adolescents with obesity/morbid obesity (mean age, $13.68 \pm 2.01$ years) and 30 healthy controls (mean age, $12.96 \pm 2.5$ years) were enrolled in this study. On pubertal assessment all participants were stage $\geq 2$, based on clinical examination and palpation. No significant difference in sex distribution was observed between the two groups $(p>0.05)$. The characteristics and baseline laboratory values of the patients and control subjects are shown in Table 1. The BMI, BMI-SDS, waist circumference, glucose, insulin, HOMA-IR, TC, triglycerides, systolic blood pressure and diastolic blood pressure were significantly higher and the neudesin level significantly lower in the patients with obesity than in the controls (Table 1). Significant differences were noted in the presence of acanthosis and IR between the patient and control groups $(p<0.01)$.

The characteristics and baseline laboratory values of the patients with obesity and morbid obesity are shown in Table 2. The BMI, BMI-SDS, insulin and HOMA-IR values were significantly higher and the neudesin level significantly lower in the patients with morbid obesity than in the patients with obesity (Table 2). No statistically significant relationships were found between these groups in terms of blood pressure, glucose or lipid levels ( $p>0.05$ ).

There was a significant correlation between neudesin and BMI-SDS (Figure 1) and the systolic and diastolic blood pressures, which were all negatively correlated $\left(r=-0.401^{* *}\right.$, $r=-0.246^{*}$ and $r=-0.250^{*}$, respectively) (Table 3). No significant correlation was found for the other parameters assessed.

\begin{tabular}{|c|c|c|c|}
\hline & $\begin{array}{l}\text { Obese patients }(n=58) \\
\text { Median }(\min -\max )\end{array}$ & $\begin{array}{l}\text { Controls }(n=30) \\
\text { Median }(\min -\max )\end{array}$ & $\mathrm{p}$ value \\
\hline Age (years) & $14.25(10.10-17.00)$ & $12.78(9.00-17.00)$ & 0.150 \\
\hline Gender (female/male) & $37 / 21$ & $16 / 14$ & 0.348 \\
\hline Height (cm) & $161.93(138.00-180.00)$ & $165.25(127.0-178.20)$ & 0.670 \\
\hline Height-SDS & $0.66(-2.15-2.97)$ & $-0.07(-1.80-2.24)$ & $0.001 * *$ \\
\hline Weight (kg) & $82.20(55.50-130.00)$ & $53.75(25.00-76.00)$ & $<0.001 * * *$ \\
\hline Weight-SDS & $3.14(1.58-6.36)$ & $0.09(-1.41-1.80)$ & $<0.001 * * *$ \\
\hline BMI $\left(\mathrm{kg} / \mathrm{m}^{2}\right)$ & $31.83(24.96-44.70)$ & $20.47(15.50-24.70)$ & $<0.001 * * *$ \\
\hline BMI-SDS & $2.73(1.35-4.27)$ & $0.18(-0.91-1.40)$ & $<0.001 * * *$ \\
\hline $\mathrm{SBP}(\mathrm{mm} / \mathrm{Hg})$ & $120.06(90-160)$ & $110.20(100-120)$ & $<0.001 * * *$ \\
\hline $\mathrm{DBP}(\mathrm{mm} / \mathrm{Hg})$ & $80.94(52-105)$ & $74.14(70-80)$ & $<0.001 * * *$ \\
\hline Waist/hip ratio & $0.87(0.69-1.02)$ & $0.83(0.78-0.89)$ & $0.026^{*}$ \\
\hline Glucose (mg/dL) & $96.33(78-118)$ & $90.33(79-97)$ & $0.001 * *$ \\
\hline Insulin $(\mu \mathrm{U} / \mathrm{mL})$ & $27.18(7.46-93.21)$ & $7.53(3.40-12.00)$ & $<0.001 * * *$ \\
\hline HOMA-IR & $6.38(1.46-26.01)$ & $1.70(0.76-2.81)$ & $<0.001 * *$ \\
\hline $\mathrm{TC}(\mathrm{mg} / \mathrm{dL})$ & $146.75(100-205)$ & $126.40(96-169)$ & $<0.001 * * *$ \\
\hline TG (mg/dL) & $95.50(33-342)$ & $72.67(44-122)$ & $0.019^{*}$ \\
\hline HDL (mg/dL) & $44.25(33-90)$ & $42.40(34-69)$ & 0.245 \\
\hline LDL (mg/dL) & $81.17(37-132)$ & $73.80(41-101)$ & 0.091 \\
\hline Neudesin (ng/mL) & $2.64(1.14-6.17)$ & $3.12(1.76-10.91)$ & $0.013 *$ \\
\hline \multicolumn{4}{|c|}{$\begin{array}{l}{ }^{*} p<0.05, * * p<0.01, * * * p<0.001 \text { and significant values are indicated in bold. } \\
\text { The data are expressed as medians and (min-max). } \\
\text { BMI-SDS: body mass index-standard deviation score, SBP: systolic blood pressure, DBP: diastolic blood pressure, HOMA-IR: homeostasis model assessment of insulin } \\
\text { resistance, TC: total cholesterol, TG: triglycerides, HDL: high-density lipoprotein, LDL: low-density lipoprotein, min-max: minimum-maximum }\end{array}$} \\
\hline
\end{tabular}


Of the 58 patients with obesity, $31 \% \quad(n=18)$ were diagnosed with MS by the IDF Consensus Worldwide definition. Interestingly, neudesin levels were higher in patients with MS compared to those without MS but the differences were not significant $(3.27 \pm 1.54$ vs $2.86 \pm 1.04$ ng/L, respectively). We also investigated neudesin levels in further subgroups stratified by the presence or absence of dyslipidemia, hypertension and IR but no significant relationships were observed (Table 4).

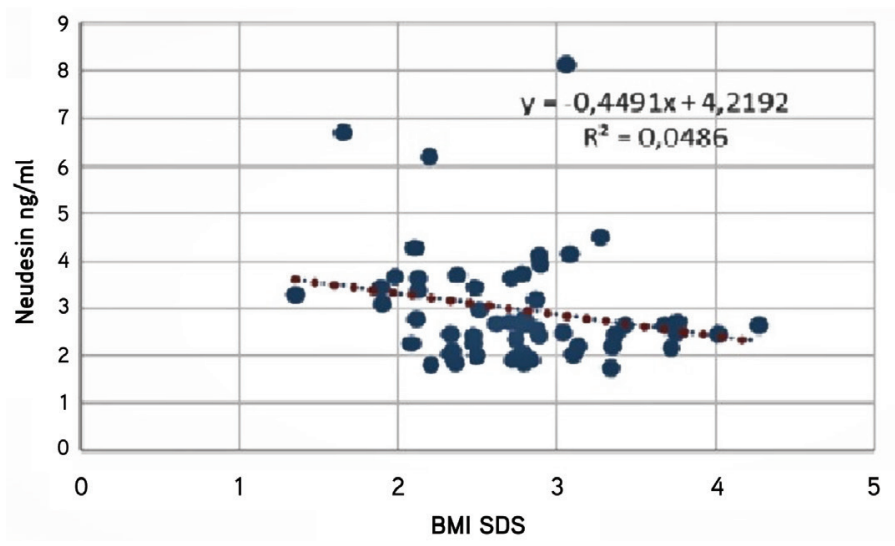

Figure 1. Correlation between neudesin and BMI-SDS

BMI-SDS: body mass index-standard deviation score

\section{Discussion}

Experimental studies suggest that neudesin may be a novel regulator of energy homeostasis and food intake, with a potential role in the development of obesity and its complications. Previously published studies reported a preferential expression of neudesin in the paraventricular nuclei and arcuate nucleus, which are important areas of the hypothalamus for regulating appetite $(14,15,16)$. It has also been proposed that neudesin may affect melanocortin signalling, as administration of recombinant neudesin in a mouse model via an intracerebrovascular cannula increased the hypothalamic expression of proopiomelanocortin (POMC) and melanocortin 4 receptor (MC4R) mRNA and decreased food intake and body weight (14). Su et al (17) showed that neudesin (NENF) acts as a negative regulator of myogenesis in bovines and that knockdown of NENF inhibited pre-adipocyte differentiation and promoted myoblast myogenesis.

In the present study, we found significantly lower neudesin levels in adolescents with obesity, as well as a negative correlation between neudesin levels and BMI, with neudesin levels decreasing as BMI-SDS increased. The specificity of serum neudesin was $83.33 \%$, with a likelihood ratio of 4.14 and diagnostic accuracy of $73.86 \%$ between healthy and obese adolescents. To the best of our knowledge, this

\begin{tabular}{|c|c|c|c|}
\hline & $\begin{array}{l}\text { Obese patients }(n=15) \\
\text { Median }(\min -\max )\end{array}$ & $\begin{array}{l}\text { Morbid obese }(n=43) \\
\text { Median }(\min -\max )\end{array}$ & $\mathrm{p}$ \\
\hline Age (years) & $14.70(10.11-17.00)$ & $14.11(10.10-16.70)$ & 0.192 \\
\hline Gender (female/male) & $10 / 5$ & $27 / 16$ & 0.793 \\
\hline Height $(\mathrm{cm})$ & $166.10(145.80-180.00)$ & $161.00(138.00-179.30)$ & 0.046 \\
\hline Height-SDS & $0.64(-2.15-2.97)$ & $0.72(-0.43-2.96)$ & 0.198 \\
\hline Weight-SDS & $2.46(1.58-3.84)$ & $3.19(1.77-6.36)$ & 0.005 \\
\hline BMI $\left(\mathrm{kg} / \mathrm{m}^{2}\right)$ & $27.87(24.96-32.00)$ & $33.90(27.10-44.70)$ & $<0.001$ \\
\hline BMI-SDS & $2.10(1.35-2.51)$ & $2.83(2.08-4.27)$ & $<0.001$ \\
\hline $\mathrm{SBP}(\mathrm{mm} / \mathrm{Hg})$ & $120.00(100-133)$ & $120.00(90-160)$ & 0.201 \\
\hline DBP (mm/Hg) & $80.00(65-90)$ & $81.00(52-105)$ & 0.631 \\
\hline HOMA-IR & $4.58(2.81-7.36)$ & $6.94(1.46-26.01)$ & 0.029 \\
\hline $\mathrm{TC}(\mathrm{mg} / \mathrm{dL})$ & $162.00(107-205)$ & $144.00(100-204)$ & 0.482 \\
\hline TG (mg/dL) & $81.00(45-157)$ & $96.00(33-342)$ & 0.597 \\
\hline HDL-C (mg/dL) & $45.00(35-90)$ & $44.00(33-74)$ & 0.289 \\
\hline LDL-C (mg/dL) & $81.00(48-106)$ & $81.00(37-132)$ & 0.791 \\
\hline Neudesin (ng/mL) & $3.19(1.714-6.177)$ & $2.47(1.144-4.502)$ & 0.043 \\
\hline
\end{tabular}

Significant values are indicated in bold.

The data are expressed as medians and (min-max).

BMI-SDS: body mass index-standard deviation score, SBP: systolic blood pressure, DBP: diastolic blood pressure, HOMA-IR: homeostasis model assessment of insulin resistance, TC: total cholesterol, TG: triglycerides, HDL-C: high-density lipoprotein-cholesterol, LDL-C: low-density lipoprotein-cholesterol, min-max: minimum-maximum 
is the first description of a relationship between obesity, metabolic complications and neudesin levels in children. Very few studies have reported a similar correlation between neudesin and BMI in adults and animal models $(3,18)$.

Bozkaya et al (19) found that circulating neudesin levels were decreased in subjects with polycystic ovary syndrome (PCOS) compared to controls. They proposed, based on their BMI data, that neudesin levels were notably lower in overweight and obese subjects than in lean subjects in both the PCOS and control groups. The work of Byerly et al (14), in their experimental study of recombinant neudesin administration showing increased hypothalamic POMC and MC4R mRNA expression and decreased food intake, suggests that neudesin acts as an anorexigenic neurotrophic factor.

Kratochvilova et al (20) also investigated serum neudesin levels and its mRNA expression in subcutaneous and visceral adipose tissue in obese subjects with or without type 2 diabetes. They found that serum neudesin concentrations in obese subjects, both with and without type 2 diabetes, did not differ from healthy lean control subjects. Similarly, Polkowska et al (21) assessed the serum levels of neudesin in children with type 1 diabetes and showed a statistically significant correlation between BMI and the levels of neudesin in the diabetic children with the longest disease duration. They found statistically higher mean neudesin concentrations in diabetic patients and proposed that IR was greater in these children with diabetes than in their healthy peers and that this difference may explain why neudesin levels were higher in patients with type 1 diabetes than non-diabetic children.

Ohta et al (3) examined the physiological role of neudesin in various tissues of wildtype mice fed a high-fat diet or normal chow food. They showed decreased food intake by

\begin{tabular}{|c|c|c|c|c|c|c|c|c|c|c|c|c|}
\hline & & BMI-SDS & $\begin{array}{l}\text { Neudesin } \\
\text { (ng/mL) }\end{array}$ & $\begin{array}{l}\text { Waist/hip } \\
\text { ratio }\end{array}$ & $\begin{array}{l}\text { Glucose } \\
\text { (mg/dL) }\end{array}$ & $\begin{array}{l}\text { Insulin } \\
(\mu \mathrm{U} / \mathrm{mL})\end{array}$ & $\begin{array}{l}\mathrm{TC} \\
(\mathrm{mg} / \mathrm{dL})\end{array}$ & $\begin{array}{l}\text { TG } \\
(\mathrm{mg} / \mathrm{dL})\end{array}$ & $\begin{array}{l}\text { HDL-C } \\
(\mathrm{mg} / \mathrm{dL})\end{array}$ & $\begin{array}{l}\text { LDL-C } \\
(\mathrm{mg} / \mathrm{dL})\end{array}$ & $\begin{array}{l}\text { SBP } \\
(\mathrm{mm} / \mathrm{Hg})\end{array}$ & $\begin{array}{l}\text { DBP } \\
(\mathrm{mm} / \mathrm{Hg})\end{array}$ \\
\hline BMI-SDS & & 1 & & & & & & & & & & \\
\hline \multirow{2}{*}{$\begin{array}{l}\text { Neudesin } \\
\text { (ng/mL) }\end{array}$} & $\mathrm{r}$ & $-0.401 * *$ & 1 & & & & & & & & & \\
\hline & $\mathrm{p}$ & $<0.001$ & & & & & & & & & & \\
\hline \multirow[t]{2}{*}{ Waist/hip ratio } & $\mathrm{r}$ & $0.293 * *$ & -0.042 & 1 & & & & & & & & \\
\hline & $\mathrm{p}$ & 0.006 & 0.705 & & & & & & & & & \\
\hline \multirow{2}{*}{$\begin{array}{l}\text { Glucose } \\
\text { (mg/dL) }\end{array}$} & $r$ & $0.284^{* *}$ & -0.073 & 0.092 & 1 & & & & & & & \\
\hline & $\mathrm{p}$ & 0.007 & 0.511 & 0.395 & & & & & & & & \\
\hline \multirow{2}{*}{$\begin{array}{l}\text { Insulin } \\
(\mu \mathrm{U} / \mathrm{mL})\end{array}$} & r & $0.634 * *$ & $-0.228^{*}$ & $0.258^{*}$ & $0.497 * *$ & 1 & & & & & & \\
\hline & $\mathrm{p}$ & $<0.001$ & 0.037 & 0.015 & $<0.001$ & & & & & & & \\
\hline \multirow{2}{*}{$\begin{array}{l}\mathrm{TC} \\
(\mathrm{mg} / \mathrm{dL})\end{array}$} & r & $0.317 * *$ & -0.090 & 0.120 & 0.195 & 0.185 & 1 & & & & & \\
\hline & $\mathrm{p}$ & 0.003 & 0.415 & 0.265 & 0.069 & 0.085 & & & & & & \\
\hline \multirow{2}{*}{$\begin{array}{l}\mathrm{TG} \\
(\mathrm{mg} / \mathrm{dL})\end{array}$} & $r$ & $0.283 * *$ & 0.040 & 0.198 & -0.041 & $0.298^{* *}$ & $0.230^{*}$ & 1 & & & & \\
\hline & $\mathrm{p}$ & 0.008 & 0.720 & 0.064 & 0.703 & 0.005 & 0.031 & & & & & \\
\hline \multirow{2}{*}{$\begin{array}{l}\text { HDL-C } \\
(\mathrm{mg} / \mathrm{dL})\end{array}$} & r & 0.032 & -0.034 & -0.033 & -0.012 & -0.168 & $0.333 *$ * & $-0.296 * *$ & 1 & & & \\
\hline & $\mathrm{p}$ & 0.766 & 0.760 & 0.760 & 0.915 & 0.118 & 0.002 & 0.005 & & & & \\
\hline \multirow{2}{*}{$\begin{array}{l}\mathrm{LDL}-\mathrm{C} \\
(\mathrm{mg} / \mathrm{dL})\end{array}$} & $r$ & 0.173 & -0.071 & -0.033 & 0.196 & 0.085 & $0.743 *$ * & 0.185 & -0.029 & 1 & & \\
\hline & $\mathrm{p}$ & 0.108 & 0.520 & 0.757 & 0.067 & 0.431 & $<0.001$ & 0.084 & 0.786 & & & \\
\hline \multirow[t]{2}{*}{$\begin{array}{l}\text { SBP } \\
(\mathrm{mm} / \mathrm{Hg})\end{array}$} & $\mathrm{r}$ & $0.474 *$ * & $-0.246^{*}$ & 0.089 & $0.357^{* *}$ & $0.430 * *$ & 0.058 & $0.214^{*}$ & -0.078 & 0.042 & 1 & \\
\hline & $\mathrm{p}$ & $<0.001$ & 0.024 & 0.409 & 0.001 & $<0.001$ & 0.592 & 0.045 & 0.472 & 0.695 & & \\
\hline \multirow[t]{2}{*}{$\begin{array}{l}\text { DBP } \\
(\mathrm{mm} / \mathrm{Hg})\end{array}$} & $r$ & $0.461 * *$ & $-0.250^{*}$ & 0.187 & $0.241 *$ & $0.442 * *$ & $0.996 * *$ & $0.297 * *$ & -0.043 & -0.028 & $0.509 * *$ & 1 \\
\hline & $\mathrm{p}$ & $<0.001$ & 0.022 & 0.082 & 0.024 & $<0.001$ & $<0.001$ & 0.005 & 0.690 & 0.797 & $<0.001$ & \\
\hline \multicolumn{13}{|c|}{$\begin{array}{l}\text { Significant values are indicated in bold. } \\
{ }^{*} \text { Correlation is significant at the } 0.05 \text { level (two-tailed). } \\
{ }^{* *} \text { Correlation is significant at the } 0.01 \text { level (two-tailed). }\end{array}$} \\
\hline
\end{tabular}




\begin{tabular}{|c|c|c|c|}
\hline & Waist/hip ratio $>90$ percentile $(n=29)$ & Waist/hip ratio $<90$ percentile $(n=26)$ & $\mathrm{p}$ value \\
\hline \multirow[t]{2}{*}{ Neudesin (ng/mL) } & $2.63(1.14-4.50)$ & $2.62(1.71-6.17)$ & 0.745 \\
\hline & Hypertensive $(n=25)$ & Non-hypertensive $(n=33)$ & \\
\hline \multirow[t]{2}{*}{ Neudesin (ng/mL) } & $2.64(1.14-4.50)$ & $2.46(1.35-6.17)$ & 0.523 \\
\hline & Insulin resistance $(n=43)$ & Non-insulin resistance $(n=15)$ & \\
\hline \multirow[t]{2}{*}{ Neudesin (ng/mL) } & $2.64(1.14-6.17)$ & $2.40(1.35-4.15)$ & 0.246 \\
\hline & Impaired fasting glucose $(n=24)$ & Non-impaired fasting glucose $(n=34)$ & \\
\hline \multirow[t]{2}{*}{ Neudesin (ng/mL) } & $2.47(1.14-4.50)$ & $2.64(1.35-6.17)$ & 0.730 \\
\hline & Dyslipidemia $(\mathrm{n}=18)$ & Non-dyslipidemia $(n=40)$ & \\
\hline \multirow[t]{2}{*}{ Neudesin (ng/mL) } & $2.64(1.35-6.17)$ & $2.43(1.14-4.10)$ & 0.379 \\
\hline & MS $(n=18)$ & Non-MS $(n=40)$ & \\
\hline Neudesin (ng/mL) & $2.64(1.14-4.50)$ & $2.52(1.35-6.17)$ & 0.259 \\
\hline
\end{tabular}

neudesin knockout mice fed normal chow, suggesting that neudesin increased food intake. By contrast, the food intake was similar between wildtype and neudesin knockout mice when fed a high-fat diet, showing that resistance to diet-induced obesity in neudesin knockout mice was independent of food intake. Neudesin administration might therefore only be efficacious in lean mice, while its effects on food intake are blunted in obese mice fed a high-fat diet. Ohta et al (3) also suggested that high fat diet-induced adipocyte hypertrophy was significantly suppressed in neudesin knockout mice. The weights of the liver and BAT were significantly smaller in neudesin knockout mice fed a high-fat diet, and they proposed that these changes might be attributed to increased sympathetic nerve activity (14).

In our study group, 18 obese children were diagnosed with MS, and this prevalence of MS was similar to previous reports from our country $(22,23)$. Neudesin levels tended to be higher in patients with MS; however, the levels were statistically comparable between obese children with or without MS. Triglyceride levels, TC levels, the HOMA-IR index and systolic and diastolic blood pressures were significantly higher in our obese group, but no statistically significant relationships were found between the neudesin levels and IR, dyslipidemia, or hypertension subgroups. In addition, neudesin levels were not different when stratified by waist/ hip ratio. Waist/hip ratio was significantly greater in our obese adolescent group compared to the healthy control group, but not strikingly so. In fact, since waist/hip ratio did not show any difference between obese and morbidly obese groups, no significant difference could be found also in neudesin levels. We found no previous studies on neudesin levels in obese children with which to compare our findings.
The study performed by Ohta (18) showed that neudesin knockout mice were protected from obesityinduced metabolic dysfunction and exhibited increased energy expenditure due to increased sympathetic activity. This, in turn, resulted in increased heat production and fatty acid oxidation in BAT and enhanced lipolysis in WAT. They proposed that glucose tolerance was impaired and insulin sensitivity was aggravated by a high-fat diet, whereas neudesin knockout mice were protected from IR induced by a high-fat diet (3).

Kratochvilova et al (20) also reported a possible role of neudesin in the development of obesity-related metabolic disturbances, based on the observed effects of acute fasting and selected weight-reducing interventions, like endoscopic duodenojejunal bypass liner implantation or gastric plication. An obese diabetic group undergoing duodenojejunal bypass liner implantation showed significantly increased concentrations of circulating neudesin. Their serum neudesin levels were also positively correlated with insulin levels and inversely with BMI, while BMI and insulin levels were correlated in subjects undergoing acute fasting.

\section{Study Limitations}

There were some limitations in our study. IR was derived by calculation rather than by insulin clamp technique, the gold standard but an invasive method. In addition, we examined the relationship between neudesin levels and metabolic outcomes. However, we could not assess its possible significance in body weight and metabolic control after weight reducing interventions. 


\section{Conclusion}

In conclusion, the serum neudesin levels were low in obese and morbidly obese adolescents and tended to increase with the appearance of metabolic disorders. These findings suggest that neudesin may play a role in the regulation of adipose tissue or energy metabolism. However, the potential role of neudesin in MS-related disorders appears to be limited, based on the findings of this study. More research is needed for a better and more robust understanding of the role of the neudesin molecule in the regulatory mechanisms in obesity and related metabolic disorders.

\section{Ethics}

Ethics Committee Approval: The study was approved by the Tekirdağ Namık Kemal University University of NonInvasive Clinical Research Ethics Committee (protocol number: 2019.137.08.09, date: 01.08.2019).

Informed Consent: Written informed consent was obtained from the children's parents after they were informed of the aim and procedures of the study.

Peer-review: Externally peer-reviewed.

\section{Authorship Contributions}

Surgical and Medical Practices: Çiğdem Binay, Concept: Aliye Çelikkol, Çiğdem Binay, Designn: Aliye Çelikkol, Çiğdem Binay, Savaş Güzel, Data Collection or Processing: Aliye Çelikkol, Çiğdem Binay, Özge Ayçiçek, Savaş Güzel, Analysis or Interpretation: Aliye Çelikkol, Çiğdem Binay, Özge Ayçiçek, Savaş Güzel, Literature Search: Aliye Çelikkol, Çiğdem Binay, Özge Ayçiçek, Savaş Güzel, Writing: Aliye Çelikkol, Çiğdem Binay, Savaş Güzel.

Financial Disclosure: The authors declared that this study received no financial support.

\section{References}

1. Sabin MA, Shield JPH. Childhood obesity. Front Horm Res 2008;36:85-96.

2. Snitker S, Macdonald I, Ravussin E, Astrup A. The sympathetic nervous system and obesity: role in aetiology and treatment. Obes Rev 2000; $1: 5-15$

3. Ohta H, Konishi M, Kobayashi Y, Kashio A, Mochiyama T, Matsumura S, Inoue K, Fushiki T, Nakao K, Kimura I, Itoh N. Deletion of the Neurotrophic Factor neudesin Prevents Diet-induced Obesity by Increased Sympathetic Activity. Sci Rep 2015;5:10049.

4. Dawbarn D, Allen SJ. Neurotrophins and neurodegeneration. Neuropathol Appl Neurobiol 2003;29:211-230.

5. Ernsberger $U$. Role of neurotrophin signalling in the differentiation of neurons from dorsal root ganglia and sympathetic ganglia. Cell Tissue Res 2009;336:349-384. Epub 2009 Apr 23

6. Jahed A, Kawaja MD. The influences of p75 neurotrophin receptor and brain-derived neurotrophic factor in the sympathetic innervation of target tissues during murine postnatal development. Auton Neurosci 2005; $118: 32-42$.

7. Ohta H, Kimura I, Konishi M, Itoh N. Neudesin as a unique secreted protein with multi-functional roles in neural functions, energy metabolism, and tumorigenesis. Front Mol Biosci 2015;2:24.

8. Marshall WA, Tanner JM. Variations in pattern of pubertal changes in girls. Arch Dis Child 1969;44:291-303

9. Zimmet P, Alberti KG, Kaufman F, Tajima N, Silink M, Arslanian S, Wong G, Bennett P, Shaw J, Caprio S; IDF Consensus Group. The metabolic syndrome in children and adolescents - an IDF consensus report. Pediatr Diabetes 2007;8:299-306.

10. Neyzi O, Furman A, Bundak R, Gunoz H, Darendeliler F, Bas F. Growth references for Turkish children aged 6 to 18 years. Acta Paediatr 2006;95:1635-1641.

11. Barlow SE; Expert Committee. Expert committee recommendations regarding the prevention, assessment, and treatment of child and adolescent overweight and obesity: summary report. Pediatrics 2007;120(Suppl 4):164-192.

12. National High Blood Pressure Education Program Working Group on High Blood Pressure in Children and Adolescents. The fourth report on the diagnosis, evaluation, and treatment of high blood pressure in children and adolescents. Pediatrics 2004;114(2 Suppl):555-576.

13. Kurtoğlu S, Hatipoğlu N, Mazıcıoğlu M, Kendirici M, Keskin M, Kondolot M. Insulin resistance in obese children and adolescents: HOMA-IR cutoff levels in the prepubertal and pubertal periods. J Clin Res Pediatr Endocrinol 2010;2:100-106. Epub 2010 Aug 2

14. Byerly MS, Swanson RD, Semsarzadeh NN, McCulloh PS, Kwon K, Aja S, Moran TH, Wong GW, Blackshaw S. Identification of hypothalamic neuron-derived neurotrophic factor as a novel factor modulating appetite. Am J Physiol Regul Integr Comp Physiol 2013;304:10851095. Epub 2013 Apr 10

15. Schwartz MW, Woods SC, Porte D Jr, Seeley RJ, Baskin DG. Central nervous system control of food intake. Nature 2000;404:661-671.

16. Münzberg H. Leptin-signaling pathways and leptin resistance. Forum Nutr 2010;63:123-132. Epub 2009 Nov 27

17. Su X, Wang Y, Li A, Zan L, Wang H. Neudesin Neurotrophic Factor Promotes Bovine Preadipocyte Differentiation and Inhibits Myoblast Myogenesis. Animals (Basel) 2019;9:1109.

18. Ohta H. Role of the secretory protein neudesin in energy metabolism. J Phys Fitness Sports Med 2016;5:229-233.

19. Bozkaya G, Fenercioglu O, Demir İ, Guler A, Aslanipour B, Calan M. Neudesin: a neuropeptide hormone decreased in subjects with polycystic ovary syndrome. Gynecol Endocrinol 2020;36:849-853. Epub 2020 Apr 21

20. Kratochvilova H, Lacinova Z, Klouckova J, Kavalkova P, Cinkajzlova A, Trachta P, Krizova J, Benes M, Dolezalova K, Fried M, Vlasakova Z, Pelikanova T, Spicak J, Mraz M, Haluzik M. Neudesin in obesity and type 2 diabetes mellitus: the effect of acute fasting and weight reducing interventions. Diabetes Metab Syndr Obes 2019;12:423-430.

21. Polkowska A, Pasierowska IE, Pasławska M, Pawluczuk E, Bossowski A. Assessment of Serum Concentrations of Adropin, Afamin, and Neudesin in Children with Type 1 Diabetes. Biomed Res Int 2019;2019:6128410.

22. Özgen Sarı İ, Boğa A, Karacı M. Evaluatıon of Frequency of Metabolıc Syndrome In Obese Children. Haydarpasa Numune Med J 2019;59:256260.

23. Binay Ç, Paketçi C, Güzel S, Samancı N. Serum irisin and oxytocin levels as predictors of metabolic parameters in obese children. J Clin Res Pediatr Endocrinol 2017;9:124-131. Epub 2017 Jan 12 